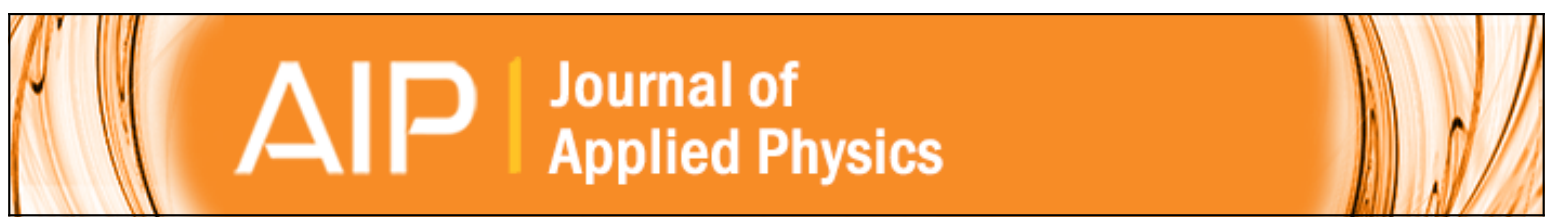

\title{
Observation of the 169Tm and 27AI NMR in Thulium Aluminum Garnet
}

E. D. Jones and V. H. Schmidt

Citation: Journal of Applied Physics 40, 1406 (1969); doi: 10.1063/1.1657691

View online: http://dx.doi.org/10.1063/1.1657691

View Table of Contents: http://scitation.aip.org/content/aip/journal/jap/40/3?ver=pdfcov

Published by the AIP Publishing

\section{Articles you may be interested in}

Deep ultraviolet photoluminescence of Tm-doped AIGaN alloys

Appl. Phys. Lett. 94, 111103 (2009); 10.1063/1.3097808

Dynamics of ultraviolet emissions in Tm-doped AIN using above band gap excitation Appl. Phys. Lett. 93, 061110 (2008); 10.1063/1.2970993

Correlation between compositional fluctuation and magnetic properties of Tm-doped AIGaN alloys Appl. Phys. Lett. 91, 222503 (2007); 10.1063/1.2817741

Neutron Scattering Cross Section Measurements for 169Tm via the $\left(n, n^{\prime}\right)$ Technique AIP Conf. Proc. 769, 1054 (2005); 10.1063/1.1945188

Cr:Er:Tm:Ho:yttrium aluminum garnet laser exhibiting dual wavelength lasing at 2.1 and $2.9 \mu \mathrm{m}$ : Spectroscopy and laser performance

J. Appl. Phys. 91, 11 (2002); 10.1063/1.1419211
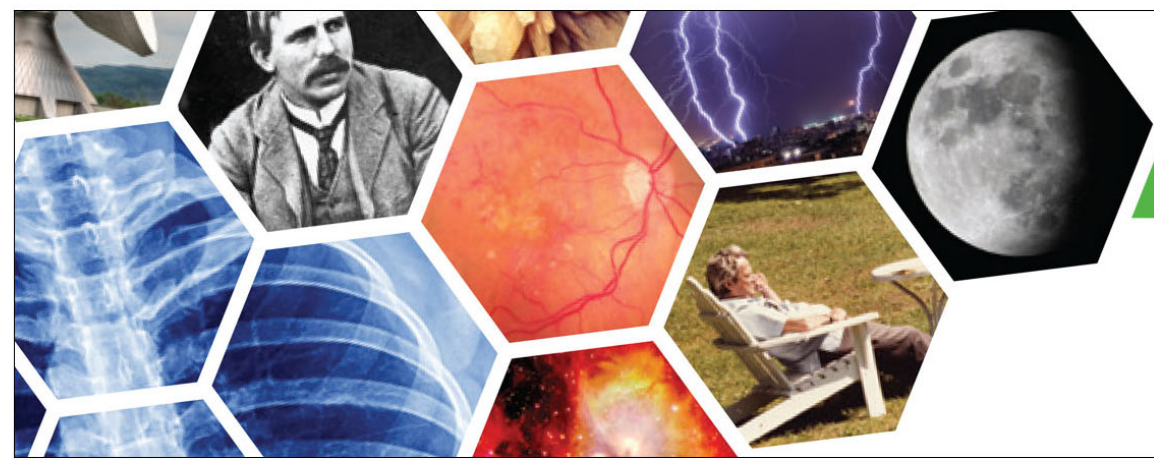


\title{
Observation of the ${ }^{169} \mathrm{Tm}$ and ${ }^{27} \mathrm{Al}$ NMR in Thulium Aluminum Garnet*
}

\author{
E. D. Jones AND V. H. SCHMIDT $\dagger$ \\ Sandia Laboratories, Albuquerque, New Mexico
}

\begin{abstract}
The observations of the ${ }^{169} \mathrm{Tm}$ and ${ }^{27} \mathrm{Al}$ nuclear magnetic resonances (NMR) in a single crystal of cubic thulium aluminum garnet are reported. The ${ }^{27} \mathrm{Al} \mathrm{MNR}$ was studied between $1.5^{\circ}$ and $300^{\circ} \mathrm{K}$, while the ${ }^{169} \mathrm{Tm}$ NMR was observed only for $T<4^{\circ} \mathrm{K}$. From a study of the angular dependences of the ${ }^{169} \mathrm{Tm}$ NMR fields, the $(\xi, \eta, \zeta)$ components of the $\mathrm{Tm}^{3+}$ susceptibility tensor were found to be highly ansiotropic and in units of emu/gram-atom, $\chi_{1}=0.014, \chi_{2}=0.551$, and $\chi_{3}=0.03$. The room temperature ${ }^{27} \mathrm{Al}$ nuclear quadrupolar coupling constants $e^{2} q Q / h$ for the $a$ and $d$ sites were measured to be $(0.892 \pm 0.005)$ and $(6.155 \pm$ $0.005) \mathrm{MHz}$, respectively. The ${ }^{27} \mathrm{Al}$ NMR frequency shifts for both sites were found to be angular and temperature dependent. A calculation for the observed angular dependences of the ${ }^{27} \mathrm{Al}$ NMR frequency shifts in terms of dipolar fields is found to give reasonable agreement with experiment for the $1.5^{\circ}$ and $300^{\circ} \mathrm{K}$ NMR data.
\end{abstract}

The recent observations ${ }^{1-4}$ of the ${ }^{169} \mathrm{Tm}$ nuclear magnetic resonance (NMR) in thulium compounds where a singlet (or nonmagnetic) ground state exists makes it possible to study in detail the magnitudes and angular dependences of the field-induced magnetization at each type of $\mathrm{Tm}^{3+}$ site in the unit cells for these compounds. This particular technique has been used to study thulium gallium garnet ${ }^{5}$ where it was shown that the $\mathrm{Tm}^{8+}$ crystal-field interaction has orthorhombic symmetry. Since the symmetry of the garnet lattice is cubic, conventional paramagnetic susceptibility measurements could not yield conclusive information about this orthorhombic crystal-field interaction.

In this paper we have extended these kinds of NMR measurements to thulium aluminum garnet (TmAlG). In addition to the ${ }^{169} \mathrm{Tm}$ NMR data $\left(T<4^{\circ} \mathrm{K}\right)$, the ${ }^{27} \mathrm{Al} \mathrm{NMR}$ was studied between $1.5^{\circ}$ and $300^{\circ} \mathrm{K}$. The ${ }^{27} \mathrm{Al}$ NMR paramagnetic frequency shifts and nuclear quadrupolar coupling constants were measured for both the $a$ - and $d$-aluminum sites in TmAlG as a function of temperature.

The ${ }^{169} \mathrm{Tm}$ and ${ }^{27} \mathrm{Al}$ NMR were observed in a single crystal of TmAlG using a variable frequency induction spectrometer and a conventional laboratory electromagnet. The NMR measurements were made between 2 and $26 \mathrm{MHz}$. The TmAlG single crystals were not spherical in shape; however, the demagnetization field due to the shape of the samples was found to be negligible over the entire temperature range.

The TmAlG unit cell contains six magnetically inequivalent $\mathrm{Tm}^{3+}$ sites which are related by $90^{\circ}$ rotations and reflections. A figure showing the orientations of the $\mathrm{Tm}^{3+}$ local symmetry axes $(\xi, \eta, \xi)$ for the six sites is given in Ref. 5. Also given in Ref. 5 are the direc-

* This work was supported by the U.S. Atomic Energy Commission.

$\dagger$ AWU Fellow-Montana State University.

i S. A. Al'tshuler and M. A. Teplov, Zh. Eksperim. i Teor. Fiz.-Pis'ma Redakt 5, 209 (1967) [translation: JETP Letters 5, 167 (1967)].

2 E. D. Jones, Phys. Rev. Letters 19, 432 (1967)

M. A. Teplov, Zh. Eksp. i Teor. Fiz. 53, 1510 (1967).

4 M. A. Teplov, Fiz. Tverd. Tela 10, 2548 (1968)

E. D. Jones, J. Phys. Chem. Solids 29, 1305 (1968). tions of the components of the $\mathrm{Tm}^{3+}$ susceptibility tensor $\chi_{1}, \chi_{2}$, and $\chi_{3}$.

The strength of the ${ }^{169} \mathrm{Tm}$ hyperfine interaction $A \mathbf{I}\langle\mathrm{J}\rangle$ has previously been determined ${ }^{5}$ and is $(A / h)=$ $-388.8 \mathrm{MHz}$. Thus, in terms of the $\mathrm{Tm}^{3+}$ atomic susceptibility tensor $\{x\}$, the ${ }^{169} \mathrm{Tm}$ hyperfine field $H_{\mathrm{hf}}$ is given ${ }^{5}$ by $\mathrm{H}_{\mathrm{hf}}=-\left(A / \gamma \hbar_{J} \beta\right) \mathrm{H} \cdot\{\chi\}$, where $g_{x}$ is the $\mathrm{Tm}^{3+}$ Landé $g$ factor and $|\gamma / 2 \pi|=0.346 \mathrm{kHz}$ per gauss is the ${ }^{169} \mathrm{Tm}$ nuclear gyromagnetic ratio, uncorrected for diamagnetism. Because the components $\chi_{1}, \chi_{2}$, and $\chi_{3}$ of the orthorhombic susceptibility tensor are not equal, the $\mathrm{Tm}^{3+}$ hyperfine field $H_{\mathrm{hf}}$ will not be, in general, colinear with the applied magnetic field $H_{0}$. Thus, the field for resonance was calculated from the vector sum $\mathrm{H}_{L}=\mathrm{H}_{0}+\mathrm{H}_{\mathrm{hf}}$. However, for the special case that the magnetic field $H_{0}$ is along one of the three principal axes $(\xi, \eta, \zeta)$ of the $\mathrm{Tm}^{3+}$ susceptibility tensor, the expressions for each of these ${ }^{169} \mathrm{Tm}$ NMR shifts $(\Delta H / H)$ simplify to $\left(-A / \gamma \hbar g_{J} \beta\right) \chi_{1},\left(-A / \gamma \hbar g_{J} \beta\right) \chi_{2}$, and $\left(-A / \gamma \hbar_{g_{J} \beta}\right) \chi_{3}$, respectively. ${ }^{5}$

For a rotation of the magnetic field $H_{0}$ in the [110] plane, there are at most four ${ }^{169} \mathrm{Tm}$ NMR fields $H_{0}$ and their measured angular dependences at $25 \mathrm{MHz}$ and $1.5^{\circ} \mathrm{K}$ are shown in Fig. 1. The solid lines drawn through the data shown in Fig. 1 are the calculated angular dependences using the measured NMR frequency shifts $(\Delta H / H)_{1}=2.50,(\Delta H / H)_{2}=95.34$, and $(\Delta H / H)_{3}=5.21$. Thus, with a knowledge of the hyperfine coupling constant $A$, the components $\chi_{1}, \chi_{2}$, and $\chi_{3}$ are easily calculated to be $\chi_{1}=0.014, \chi_{2}=0.551$, and $\chi_{3}=0.030$ in units of emu/gram-atom. The total paramagnetic susceptibility of the TmAlG unit cell can be calculated to be $\chi_{1}=\frac{1}{3}\left(\chi_{1}+\chi_{2}+\chi_{3}\right)=0.198 \mathrm{emu} / \mathrm{gram}-$ atom and is in good agreement with the measured value of $0.19 \mathrm{emu} / \mathrm{gram}$ atom. ${ }^{6}$

The thulium resonance is unobservable above about $20^{\circ} \mathrm{K}$, hence, additional information about the temperature dependence of the $\mathrm{Tm}^{3+}$ magnetization was obtained from ${ }^{27} \mathrm{Al}$ NMR measurements of the para-

${ }^{\circ}$ W. P. Wolf, M. Ball, M. T. Hutchings, M. J. M. Leask, and A. F. G. Wyatt, J. Phys. Soc. Japan 17, Supplement B-I, 443 (1962). 


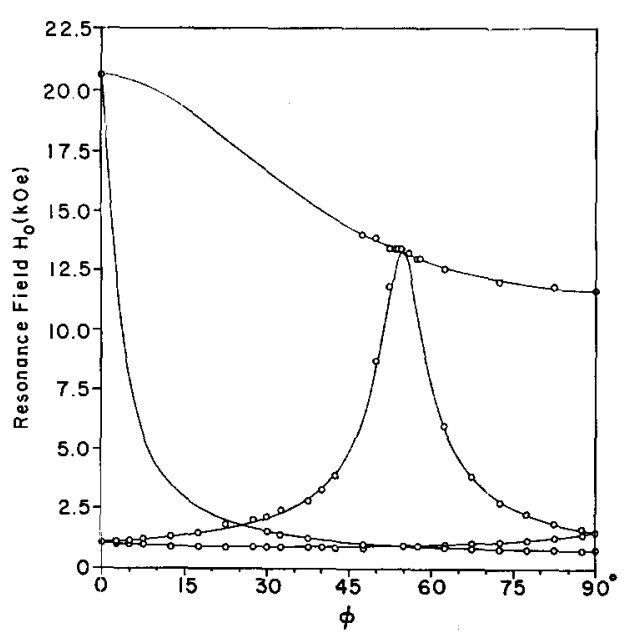

Fig. 1. The angular dependence of the ${ }^{169} \mathrm{Tm}$ NMR fields $H_{0}$ in the [110] plane for $\nu=25.000 \mathrm{MHz}$ and $T=1.5^{\circ} \mathrm{K}$. The $\langle 100\rangle$ and $\langle 110\rangle$ directions are given by $\varphi=0^{\circ}$ and $\varphi=90^{\circ}$, respectively. The three NMR fields for the $(\xi, \eta, \zeta)$ axes are, respectively, $20.658,0.750$, and $11.644 \mathrm{kOe}$. The open circles are the measured NMR data and the solid lines are the calculated values.

magnetic shift due to the thulium ions. The ${ }^{27} \mathrm{Al}$ nuclei are in $a$ and $d$ sites having axial symmetry in the $\langle 111\rangle$ and $\langle 100\rangle$ directions, respectively. Their NMR spectra were analyzed using expressions developed by Pound ${ }^{7}$ and corrected by Volkoff, ${ }^{8}$ which give terms through third order in the quadrupolar perturbation.

The measured $a$-site quadrupolar coupling constant $\left|e^{2} q Q / h\right|$ decreased from $(0.98 \pm 0.03) \mathrm{MHz}$ at $4^{\circ} \mathrm{K}$ to $(0.892 \pm 0.005) \mathrm{MHz}$ at $300^{\circ} \mathrm{K}$ while the $d$-site coupling constant apparently increased from $(6.10 \pm 0.06)$ to $(6.155 \pm 0.005) \mathrm{MHz}$ over the same temperature range. The $300^{\circ} \mathrm{K}$ values are in accord with the almost

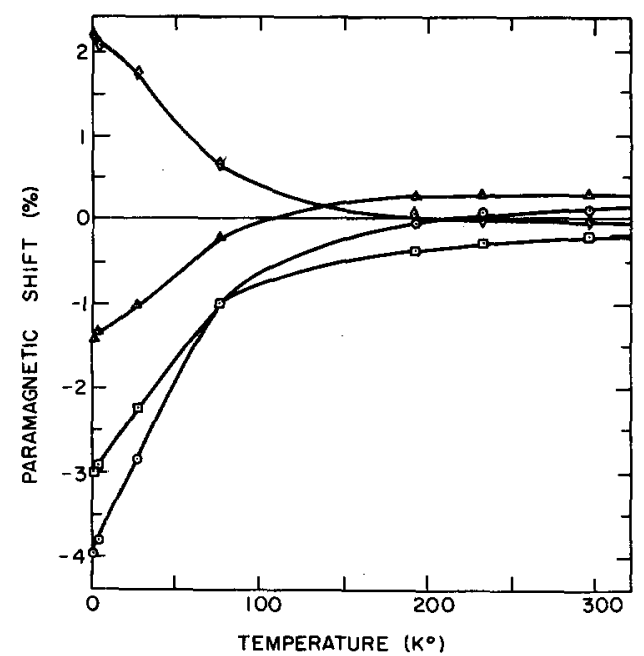

FIG. 2. The temperature dependence of the ${ }^{27} \mathrm{Al} \mathrm{NMR}$ frequency shift in TmAIG. The $a$ - and $d$-site NMR data for $H_{0}$ parallel to the site axes are labeled $\Delta$ and $O$, respectively, while the perpendicular cases are labeled $\diamond$ and $\square$.

${ }^{7}$ R. V. Pound, Phys. Rev. 79, 685 (1950).

${ }^{8}$ G. M. Volkoff, Canad. J. Phys. 31, 820 (1953). linear increase in magnitude of coupling constants with atomic number of the rare earth ion previously noted for the $\mathrm{Tb}, \mathrm{Dy}$, and $\mathrm{Yb}$ aluminum garnets. ${ }^{9}$

The measured paramagnetic shifts at the aluminum $a$ and $d$ sites for applied magnetic field $H_{0}$ both parallel and perpendicular to the site axes of symmetry are plotted in Fig. 2 as functions of temperature, and compared in Table I with values calculated using point dipole sums. At room temperature the shifts show some similarity to those calculated from dipole sums based on a free $\mathrm{Tm}^{3+}$ ion, although the susceptibility is still quite anisotropic, as evidenced by the sum of the parallel shift and twice the perpendicular shift being far from zero for both the $a$ and $d$ sites.

The agreement between the paramagnetic shifts measured at liquid helium temperatures and the shifts

TABLE I. ${ }^{27} \mathrm{Al}$ paramagnetic shifts for $H$ parallel and perpendicular to site axes. The shifts, calculated from thulium dipole sums at $300^{\circ}$ and $1.5^{\circ} \mathrm{K}$, are based on the free $\mathrm{Tm}^{3+}$ ion and the $1.5^{\circ} \mathrm{K}$ susceptibility tensors, respectively.

\begin{tabular}{cclcc}
\hline $\begin{array}{c}\text { Temp., } \\
{ }^{\circ} \mathrm{K}\end{array}$ & Site & $\begin{array}{c}H_{0} \\
\text { Direction }\end{array}$ & Measured & $\begin{array}{c}\text { Dipole } \\
\text { sum }\end{array}$ \\
\hline 297 & $a$ & parallel & $+0.28 \pm 0.01$ & +0.392 \\
297 & $a$ & perpendicular & $-0.02 \pm 0.02$ & -0.196 \\
297 & $d$ & parallel & $+0.012 \pm 0.01$ & +0.404 \\
297 & $d$ & perpendicular & $-0.22 \pm 0.01$ & -0.202 \\
1.5 & $a$ & parallel & $-1.41 \pm 0.20$ & -1.59 \\
1.5 & $a$ & perpendicular & $+2.16 \pm 0.10$ & +1.77 \\
1.5 & $d$ & parallel & $-3.95 \pm 0.10$ & -3.68 \\
1.5 & $d$ & perpendicular & $-3.01 \pm 0.30$ & -2.83 \\
\hline
\end{tabular}

calculated using dipole sums based on the anisotropic thulium susceptibility tensor found from the $1.5^{\circ} \mathrm{K}$ ${ }^{169} \mathrm{Tm}$ NMR data is quite good for one of the two possible assignments of directions for $\chi_{2}$ and $\chi_{3}$, but is not even qualitatively correct for the other choice. Both $\chi_{2}$ and $\chi_{3}$ are in $\langle 110\rangle$ directions perpendicular to the line joining a thulium ion and its nearest $d$-site aluminum neighbors. The good agreement occurs if the $\chi_{2}$ (easy magnetization) direction is the one more nearly parallel to the line joining the two oxygens closest to the thulium ion. The deviations of the measured shifts from the dipole sums at helium temperatures probably occur because the nearest thulium ions produce fields differing appreciably from the point dipole fields, although the possibility of a transferred hyperfine interaction is not ruled out.

The authors wish to thank R. J. Baughman for growing the single crystals and Dr. A. Narath for providing the dipole sum computer program.

D. T. Edmonds and A. J. Lindop, J. Appl. Phys. 39, 1008 (1968). 